\title{
A Numerical Integration Formula Based on the Bessel Functions
}

By

\author{
Hidenori OGATA*
}

\begin{abstract}
In this paper, we discuss the properties of a quadrature formula with the zeros of the Bessel functions as nodes for integrals $\int_{-\infty}^{\infty}|x|^{2 \nu+1} f(x) \mathrm{d} x$, where $\nu$ is a real constant greater than -1 and $f(x)$ is a function analytic on the real axis $(-\infty,+\infty)$. We show from theoretical error analysis that (i) the quadrature formula converges exponentially, (ii) it is as accurate as the trapezoidal formula over $(-\infty,+\infty)$ and (iii) the accuracy of the quadrature formula doubles that of an interpolation formula with the same nodes. Numerical examples support the above theoretical results. We also apply the quadrature formula to the numerical integration of integral involving the Bessel function.
\end{abstract}

\section{$\S 1$. Introduction}

In this paper, we investigate the quadrature formula with the zeros of the Bessel functions as nodes, namely,

$$
\int_{-\infty}|x|^{2 \nu+1} f(x) \mathrm{d} x \approx h \sum_{\substack{k=-\infty \\ k \neq 0}}^{\infty} w_{\nu k}\left|h \xi_{\nu k}\right|^{2 \nu+1} f\left(h \xi_{\nu k}\right)
$$

with

$$
w_{\nu k}=\frac{Y_{\nu}\left(\pi \xi_{\nu|k|}\right)}{J_{\nu+1}\left(\pi \xi_{\nu|k|}\right)}=\frac{2}{\pi^{2} \xi_{\nu|k|} J_{\nu+1}\left(\pi \xi_{\nu|k|}\right)}, \quad k= \pm 1, \pm 2, \ldots,
$$

Communicated by H. Okamoto. Received December 15, 2004. Revised April 7, 2005. 2000 Mathematics Subject Classification(s): 65D30

Key words: numerical integration, quadrature, Bessel function, Hankel transform

* Department of Computer Science, Faculty of Engineering, Ehime University, Matsuyama 790-8577, Japan.

current address: Department of Computer Science, Faculty of Electro-Communications, The University of Electro-Communications, Chofu 182-8585, Japan.

e-mail: ogata@im.uec.ac.jp

(C) 2005 Research Institute for Mathematical Sciences, Kyoto University. All rights reserved. 
where $\nu$ is a real constant greater than $-1, h$ is a positive constant, $\xi_{\nu k}, k=$ $\pm 1, \pm 2, \ldots$ are the zeros of the Bessel function $J_{\nu}(\pi x)$ of the first kind of order $\nu$ ordered in such a way that

$$
\cdots<\xi_{\nu-2}<\xi_{\nu-1}<0<\xi_{\nu 1}<\xi_{\nu 2}<\cdots ; \quad \xi_{\nu-k}=-\xi_{\nu k}, k=1,2, \ldots
$$

and $Y_{\nu}$ is the Bessel function of the second kind of order $\nu^{1}$.

The motivation of our study is as follows. The double exponential quadrature formulae [11], abbreviated to the DE formulae, are known as optimal quadrature formulae and efficient for various types of integrals. However, the conventional DE formulae do not work well for integrals of oscillatory functions over infinite intervals. Ooura and Mori partially overcame this weakness by inventing a new formula of DE-type for integrals of the Fourier transform type [8], that is, integrals of the form

$$
\int_{0}^{\infty} f(x) \sin x \mathrm{~d} x
$$

where $f(x)$ is a function with slow decay as $x \rightarrow+\infty$. The key idea of their formula was to choose a DE transform so that the nodes of the quadrature approach rapidly to the zeros of the function $\sin x$ and the integral can be computed with a small number of function evaluations, while the DE transforms in the conventional DE formula are chosen so that the transformed integrand function decays double exponentially. We tried to extend Ooura and Mori's DE formula to a one for integrals of the Hankel transform type, that is, integrals of the form

$$
\int_{0}^{\infty} f(x) J_{\nu}(x) \mathrm{d} x
$$

where $J_{\nu}(x)$ is the Bessel function of order $\nu$ and $f(x)$ is a function with slow decay as $x \rightarrow+\infty$. Since, as the conventional DE formula, Ooura and Mori's $\mathrm{DE}$ formula is based on the trapezoidal formula over $(-\infty,+\infty)$, i.e., a quadrature with the zeros of the sine function as nodes, we naturally expect that we can compute (1.4) by a formula with the zeros of the Bessel functions as nodes coupled with a DE transform similar to Ooura and Mori's.

In a study motivated by the above discussion, we found the quadrature formula (1.1). The formula was first presented by Frappier and Olivier [2], who obtained the formula (1.1) by a limitation procedure of the Gauss-Jacobi

\footnotetext{
${ }^{1}$ The second equality of $(1.2)$ is shown by the formula $J_{\nu}(z) Y_{\nu+1}(z)-J_{\nu+1}(z) Y_{\nu}(z)=$ $-2 /(\pi z)$ (formula 9.1 .16 in $[1]$ ).
} 
quadrature and presented classes of integrand functions for which the quadrature formula gives the exact integral values. In addition to Frappier and Olivier's results, Grozev, Rahman and Ghanem presented theorems on integrand functions for which the quadrature formula (1.1) gives the exact integral values $[3,4]$.

We investigated the quadrature formula (1.1) more thoroughly and found it very efficient in the following sense.

1. The quadrature formula (1.1) converges exponentially as the density of nodes increases if the integrand function is analytic on the real axis and satisfies some conditions. Its error is of the same order as that of the trapezoidal formula with equal mesh size $h$ over the infinite interval

$$
\int_{-\infty}^{\infty} f(x) \mathrm{d} x \approx h \sum_{k=-\infty}^{\infty} f(k h),
$$

which gives the basis of the DE formula together with the DE transform technique.

2. The quadrature formula (1.1) can be regarded as an interpolation-type one, that is, it is obtained by integrating an interpolation formula with the same nodes. We show the remarkable property that the accuracy of the quadrature formula doubles that of the interpolation formula. This property is common to the Gauss-type quadrature formulae and the trapezoidal one $(1.5)$.

3. We can apply the quadrature formula (1.1) to the computations of integrals of the Hankel transform type (1.4), which is the motivation of this study.

The contents of this paper are as follows. In Section 2, we prepare some notations for theoretical analysis and show a theorem on the quadrature error of the formula (1.1), which should be compared with the one of the trapezoidal formula (1.5). In Section 3, we show the quadrature formula (1.1) can be regarded as an interpolation-type one, that is, the quadrature formula can be obtained by integrating an interpolation one with the same nodes. Then we compare the quadrature error and the interpolation error and show that the accuracy of the quadrature formula (1.1) doubles the one of the corresponding interpolation formula, noting that it is common also to the Gauss-type formula and the trapezoidal one (1.5). In Section 5, we show an application of the quadrature formula (1.1) to the computation of integrals of the Hankel 
transform type (1.4). In Section 6, we present concluding remarks and refer to problems for future studies.

\section{$\S 2 . \quad$ Quadrature Error}

Notations. Throughout this paper, we denote the integral on the left hand side of (1.1) by $\mathcal{I}_{\nu}(f)$, i.e.,

$$
\mathcal{I}_{\nu}(f)=\int_{-\infty}^{\infty}|x|^{2 \nu+1} f(x) \mathrm{d} x
$$

and the quadrature formula on the right hand side of $(1.1)$ by $\mathcal{I}_{\nu h}(f)$, i.e.,

$$
\mathcal{I}_{\nu h}(f)=h \sum_{\substack{k=-\infty \\ k \neq 0}}^{\infty} w_{\nu k}\left|h \xi_{\nu k}\right|^{2 \nu+1} f\left(h \xi_{\nu k}\right)
$$

For theoretical error analysis presented in this paper, we prepare some notations ${ }^{2}$.

Definition 2.1. $\quad$ Let $d$ be a positive constant, $D_{d}$ be the strip domain $D_{d}=\{z \in \mathbb{C}|| \operatorname{Im} z \mid<d\}$ and $\Gamma_{d}$ be the boundary of $D_{d}$.

Let $\nu$ be a real constant such that $\nu>-1$. We denote by $\mathcal{B}(\nu, d)$ the set of the functions $f(z)$ such that

1. $f(z)$ is analytic in $D_{d}$.

2. For arbitrary $c$ such that $0<c<d$, the integral

$$
\mathcal{N}_{\nu c}(f) \equiv \int_{-\infty}^{+\infty}\left[|x+\mathrm{i} c|^{2 \nu+1}|f(x+\mathrm{i} c)|+|x-\mathrm{i} c|^{2 \nu+1}|f(x-\mathrm{i} c)|\right] \mathrm{d} x
$$

exists and, in addition, the limit $\mathcal{N}_{\nu d-0}(f) \equiv \lim _{c \uparrow d} \mathcal{N}_{\nu c}(f)$ exists and is finite.

3. For arbitrary $c$ such that $0<c<d$,

$$
\lim _{x \rightarrow \pm \infty} \int_{-c}^{c}|x+\mathrm{i} y|^{2 \nu+1}|f(x+\mathrm{i} y)| \mathrm{d} y=0 .
$$

\footnotetext{
${ }^{2}$ Henceforth we denote the set of all the integers by $\mathbb{Z}$, that of all the real numbers by $\mathbb{R}$ and that of all the complex numbers by $\mathbb{C}$.
} 
Besides we define some notations of integrals. For a function $f(z)$ defined on $D_{d}$ and for $c$ such that $0<c<d$, we denote the integral of $f(z)$ over the paths

$$
\{x-\mathrm{i} c \mid-\infty<x<+\infty\} \cup\{x+\mathrm{i} c \mid+\infty>x>-\infty\}
$$

by $\int_{\Gamma_{c}} f(z) \mathrm{d} z$, that is,

$$
\int_{\Gamma_{c}} f(z) \mathrm{d} z=\int_{-\infty}^{+\infty}[f(x-\mathrm{i} c)-f(x+\mathrm{i} c)] \mathrm{d} x .
$$

Further, the limit of $\int_{\Gamma_{c}} f(z) \mathrm{d} z$ as $c \uparrow d$ exists, we denote it by $\int_{\Gamma_{d-0}} f(z) \mathrm{d} z$ :

$$
\int_{\Gamma_{d-0}} f(z) \mathrm{d} z=\lim _{c \uparrow d} \int_{\Gamma_{c}} f(z) \mathrm{d} z=\lim _{c \uparrow d} \int_{-\infty}^{+\infty}[f(x-\mathrm{i} c)-f(x+\mathrm{i} c)] \mathrm{d} x .
$$

Error Analysis. An expression by complex integral of the quadrature error of (1.1) and its upper bound are given in the following theorem.

Theorem 2.1. $\quad$ Let $\nu>-1$ and $f(z) \in \mathcal{B}(\nu, d)$. Then, the quadrature error of (1.1) is given by the complex integral

$$
\mathcal{I}_{\nu}(f)-\mathcal{I}_{\nu h}(f)=\frac{1}{2 \pi \mathrm{i}} \int_{\Gamma_{d-0}} f(z) \Phi_{\nu h}(z) \mathrm{d} z
$$

with

$$
\Phi_{\nu h}(z)= \begin{cases}-\mathrm{i} \pi z^{2 \nu+1} H_{\nu}^{(1)}(\pi z / h) / J_{\nu}(\pi z / h) & (0 \leqq \arg z<\pi) \\ +\mathrm{i} \pi z^{2 \nu+1} H_{\nu}^{(2)}(\pi z / h) / J_{\nu}(\pi z / h) & (-\pi \leqq \arg z<0),\end{cases}
$$

where $H_{\nu}^{(1)}=J_{\nu}+\mathrm{i} Y_{\nu}$ and $H_{\nu}^{(2)}=J_{\nu}-\mathrm{i} Y_{\nu}$ are the Hankel functions of order $\nu$. Besides, an upper bound of the quadrature error (2.2) is given by the inequality

$$
\left|\mathcal{I}_{\nu}(f)-\mathcal{I}_{\nu}(f, h)\right| \leqq C_{\nu d} \mathcal{N}_{\nu d-0}(f) \exp \left(-\frac{2 \pi d}{h}\right)
$$

where $C_{\nu d}$ is a positive constant depending only on $\nu$ and $d$.

Using the terminology in [12], we call the function $\Phi_{\nu h}(z)$ of $(2.3)$ the characteristic function of the quadrature formula (1.1). Theorem 2.1 says that the quadrature error of the formula (1.1) decays exponentially with order $\mathrm{O}[\exp (-2 \pi d / h)]$ as a function of $1 / h$, which is the node density per unit length since we have

$$
h \xi_{\nu k} \sim \pm h\left(|k|-\frac{\pi \nu}{2}-\frac{\pi}{4}\right) \quad \text { as } k \rightarrow \pm \infty
$$


(formula 9.5.12 in [1]) and also corresponds to the mesh size of the trapezoidal formula over the infinite interval with equidistant nodes (1.5). Roughly speaking, the upper bound of the quadrature error (2.4) is found from the remark that

$$
\begin{array}{r}
\Phi_{\nu h}(z) \approx \pm 2 \pi \mathrm{i} z^{2 \nu+1} \exp \left( \pm \mathrm{i} 2 \pi\left(\frac{z}{h}-\frac{\nu}{2}-\frac{1}{4}\right)\right) \\
\text { if } \pm \operatorname{Im} z>0 \text { as }|\operatorname{Im} z| \text { is large, }
\end{array}
$$

which is obtained from the asymptotic expansions of the Hankel functions

$$
\begin{array}{ll}
H_{\nu}^{(1)}(z) \sim \sqrt{2 /(\pi z)} \mathrm{e}^{\mathrm{i}(z-\nu \pi / 2-\pi / 4)} & (|z| \rightarrow \infty,-\pi<\arg z<2 \pi), \\
H_{\nu}^{(2)}(z) \sim \sqrt{2 /(\pi z)} \mathrm{e}^{-\mathrm{i}(z-\nu \pi / 2-\pi / 4)} & (|z| \rightarrow \infty,-2 \pi<\arg z<\pi)
\end{array}
$$

(formulae 9.2.3 and 9.2.4 in [1]), and then

$$
\left|\Phi_{\nu h}(z)\right| \approx \pi|z|^{2 \nu+1} \exp \left(-\frac{2 \pi}{h}|\operatorname{Im} z|\right) \quad \text { as }|\operatorname{Im} z| \text { is large. }
$$

The proof of Theorem 2.1 goes as follows.

Proof. We consider the integral

$$
-\int_{C_{1}+C_{2}+C_{3}} z^{2 \nu+1} f(z) \frac{H_{\nu}^{(1)}(\pi z / h)}{2 J_{\nu}(\pi z / h)} \mathrm{d} z=\frac{1}{2 \pi \mathrm{i}} \int_{C_{1}+C_{2}+C_{3}} f(z) \Phi_{\nu h}(z) \mathrm{d} z
$$

where $C_{1}, C_{2}, C_{3}$ are the integral paths shown in Figure 1. Since the integrand function is analytic in the domain $\{z \in \mathbb{C} \mid 0<\operatorname{Im} z<d\}$, the integral path $C_{1}+C_{2}+C_{3}$ can be modified to $C_{+}$given in Figure 1 as the broken line. The integrals on $\gamma_{k}, k= \pm 1, \pm 2, \ldots$ are computed as follows. If $k>0$, we have

$$
\begin{gathered}
-\int_{\gamma_{k}} z^{2 \nu+1} f(z) \frac{H_{\nu}^{(1)}(\pi z / h)}{2 J_{\nu}(\pi z / h)}=-\pi \mathrm{i} \times\left(\text { residue at } z=h \xi_{\nu k}\right)+\mathrm{O}(\rho) \\
=-\frac{\mathrm{i} h}{2}\left(h \xi_{\nu k}\right)^{2 \nu+1} \frac{H_{\nu}^{(1)}\left(\pi \xi_{\nu k}\right)}{J_{\nu}^{\prime}\left(\pi \xi_{\nu k}\right)}+\mathrm{O}(\rho) \\
=-h\left(h \xi_{\nu k}\right)^{2 \nu+1} w_{\nu k}+\mathrm{O}(\rho) \quad \text { as } \rho \rightarrow 0,
\end{gathered}
$$

where we used the formula

$$
J_{\nu}^{\prime}(z)=\frac{\nu}{z} J_{\nu}(z)-J_{\nu+1}(z)
$$




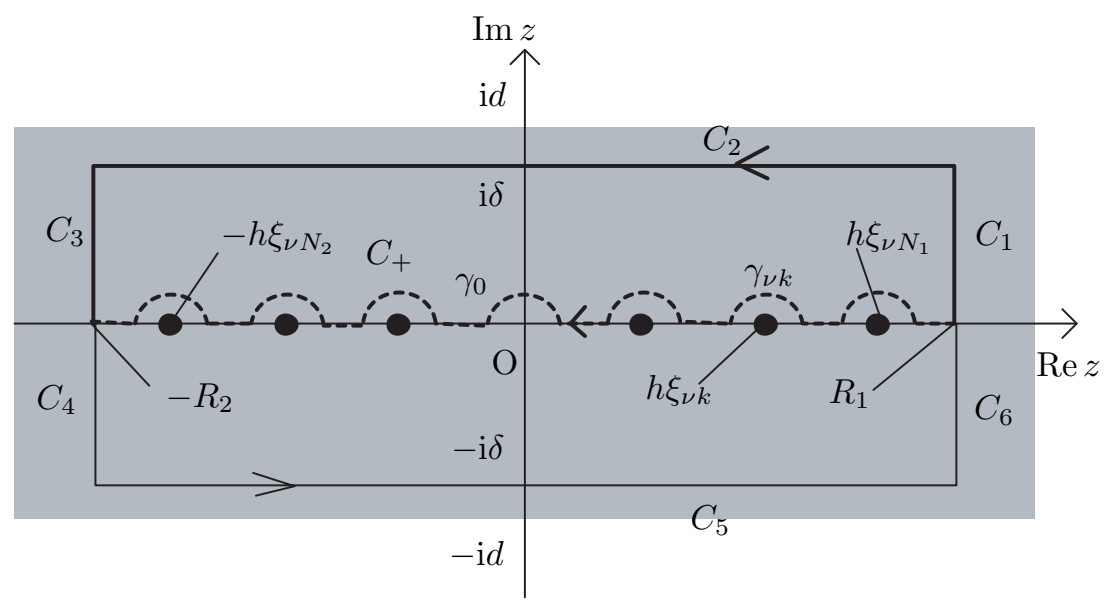

Figure 1. The integral paths $C_{1}, C_{2}, C_{3}, C_{4}, C_{5}, C_{6}$ for the proof of Theorem 2.1, where $R_{1}=h\left(N_{1}+\nu / 2+1 / 4\right)$ and $R_{2}=h\left(N_{2}+\nu / 2+1 / 4\right)$. In the proof of the theorem, the path $C_{1}+C_{2}+C_{3}$, the path $C_{1}+C_{2}+C_{3}$ is modified to the one $C_{+}$(broken line), which consists of the semi-circles $\gamma_{k}, k=-N_{2},-N_{2}+$ $1, \ldots,-1,1,2, \ldots, N_{1}$ of radius $\rho>0$ with centres at $z=h \xi_{\nu k}$, the one $\gamma_{0}$ of radius $\rho$ with centre at $z=0$, and the line segments joining them.

(formula 9.1.27 in [1]) on the third equality. If $k<0$, we have

$$
\begin{gathered}
-\int_{\gamma_{k}} z^{2 \nu+1} f(z) \frac{H_{\nu}^{(1)}(\pi z / h)}{2 J_{\nu}(\pi z / h)}=-\pi \mathrm{i} \times\left(\text { residue at } z=h \xi_{\nu k}\right)+\mathrm{O}(\rho) \\
=-\frac{\mathrm{i} h}{2}\left(-h \xi_{\nu|k|}\right)^{2 \nu+1} f\left(h \xi_{\nu k}\right) \frac{H_{\nu}^{(1)}\left(-\pi \xi_{\nu|k|}\right)}{J_{\nu}^{\prime}\left(-\pi \xi_{\nu|k|}\right)}+\mathrm{O}(\rho) \\
=\frac{\mathrm{i} h}{2}\left(\mathrm{e}^{\mathrm{i} \pi}\right)^{2 \nu+1}\left(h \xi_{\nu|k|}\right)^{2 \nu+1} f\left(h \xi_{\nu k}\right) \frac{H_{\nu}^{(1)}\left(\mathrm{e}^{\mathrm{i} \pi} \pi \xi_{\nu|k|}\right)}{J_{\nu+1}\left(\mathrm{e}^{\mathrm{i} \pi} \pi \xi_{\nu|k|}\right)}+\mathrm{O}(\rho) \\
=-\frac{h}{2} w_{\nu k}\left(h \xi_{\nu|k|}\right)^{2 \nu+1} f\left(h \xi_{\nu k}\right)+\mathrm{O}(\rho) \quad \text { as } \rho \rightarrow 0
\end{gathered}
$$

where we used the formula (2.8) on the third equality and the relations

$$
J_{\nu+1}\left(\mathrm{e}^{\mathrm{i} \pi} z\right)=\mathrm{e}^{\mathrm{i} \nu \pi} J_{\nu}(z), \quad H_{\nu}^{(1)}\left(\mathrm{e}^{\mathrm{i} \pi} z\right)=-\mathrm{e}^{-\mathrm{i} \nu \pi} H_{\nu}^{(2)}(z)
$$

(formulae 9.1.35 and 9.1.39 in [1]) on the fourth equality. The integral on $\gamma_{0}$ 
vanishes as $\rho \rightarrow 0$ since we have

$$
\frac{z^{2 \nu+1} H_{\nu}^{(1)}(\pi z / h)}{J_{\nu}(\pi z / h)}= \begin{cases}\mathrm{O}(z) & \text { if } z \notin \mathbb{Z} \\ \mathrm{O}\left(z^{2 \nu+1} \log z\right) & \text { if } z \in \mathbb{Z}\end{cases}
$$

which is obtained from the formulae $Y_{\nu}(z)=\left[J_{\nu}(z) \cos (\nu \pi)-J_{-\nu}(z)\right] / \sin (\nu \pi)$ if $\nu \notin \mathbb{Z}$ (formula 9.1.2 in [1]) and

$$
\begin{aligned}
& Y_{\nu}(z)=-\frac{(z / 2)^{-\nu}}{\pi} \sum_{k=0}^{\nu-1} \frac{(\nu-k-1) !}{k !}\left(\frac{z}{2}\right)^{2 k} \\
& +\frac{2}{\pi} \log \left(\frac{z}{2}\right) J_{\nu}(z)-\frac{(z / 2)^{\nu}}{\pi} \sum_{k=0}^{\infty}\{\psi(k+1)+\psi(\nu+k+1)\} \frac{\left(-z^{2} / 4\right)^{k}}{k !(\nu+k) !} \quad \text { if } \nu \in \mathbb{Z}
\end{aligned}
$$

with $\psi(1)=-\gamma, \psi(n)=-\gamma+\sum_{k=1}^{n-1} k^{-1}, n=2,3, \ldots(\text { formula } 9.1 .11 \text { in [1] })^{3}$. Therefore we have

$$
\begin{aligned}
& \frac{1}{2 \pi \mathrm{i}} \int_{C_{1}+C_{2}+C_{3}} f(z) \Phi_{\nu h}(z) \mathrm{d} z=\frac{1}{2} \mathrm{p} \cdot \mathrm{v} \cdot \int_{-R_{2}}^{0}|x|^{2 \nu+1} f(x) \frac{H_{\nu}^{(2)}(\pi x / h)}{J_{\nu}(\pi x / h)} \mathrm{d} x \\
& +\frac{1}{2} \mathrm{p} . \mathrm{v} \cdot \int_{0}^{R_{1}}|x|^{2 \nu+1} f(x) \frac{H_{\nu}^{(1)}(\pi x / h)}{J_{\nu}(\pi x / h)} \mathrm{d} x-\frac{h}{2} \sum_{\substack{k=-N_{2} \\
k \neq 0}}^{N_{1}} w_{\nu k}\left|h \xi_{\nu k}\right|^{2 \nu+1} f\left(h \xi_{\nu k}\right),
\end{aligned}
$$

where "p.v." denotes the principal value and we used the relations (2.9) to derive the first term of the right-hand side. Similarly we have

$$
\begin{aligned}
& \frac{1}{2 \pi \mathrm{i}} \int_{C_{4}+C_{5}+C_{6}} f(z) \Phi_{\nu h}(z) \mathrm{d} z=\frac{1}{2} \mathrm{p} \cdot \mathrm{v} \cdot \int_{0}^{R_{1}}|x|^{2 \nu+1} f(x) \frac{H_{\nu}^{(1)}(\pi x / h)}{J_{\nu}(\pi x / h)} \mathrm{d} x \\
& +\frac{1}{2} \text { p.v. } \int_{0}^{R_{1}}|x|^{2 \nu+1} f(x) \frac{H_{\nu}^{(2)}(\pi x / h)}{J_{\nu}(\pi x / h)} \mathrm{d} x-\frac{h}{2} \sum_{\substack{k=-N_{2} \\
k \neq 0}}^{N_{1}} w_{\nu k}\left|h \xi_{\nu k}\right|^{2 \nu+1} f\left(h \xi_{\nu k}\right) .
\end{aligned}
$$

\footnotetext{
${ }^{3} \gamma$ denotes Euler's constant $\gamma=0.57721566490153 \ldots$
} 
Summing (2.10) and (2.11) leads us to the equality

$$
\begin{aligned}
& \frac{1}{2 \pi \mathrm{i}} \int_{C_{1}+\cdots+C_{6}} f(z) \Phi_{\nu h}(z) \mathrm{d} z \\
& \quad=\int_{-R_{2}}^{R_{1}}|x|^{2 \nu+1} f(x) \mathrm{d} x-h \sum_{\substack{k=-N_{2} \\
k \neq 0}}^{N_{1}} w_{\nu k}\left|h \xi_{\nu k}\right|^{2 \nu+1} f\left(h \xi_{\nu k}\right) .
\end{aligned}
$$

It is shown from (2.1) and Lemma 2.2, which is given at the end of this section, that the integrals on $C_{6}+C_{1}$ and $C_{3}+C_{4}$ vanish as $N_{1}, N_{2} \rightarrow \infty$. Consequently, we obtain the equality $(2.2)$.

The inequality (2.4) is obtained by evaluating the absolute value of the complex integral on the left-hand side of (2.12) as follows. From (2.6) and Lemma 2.2 given in the last of this section, we have $\left|\Phi_{\nu h}(z)\right| \leqq 2 \pi \kappa_{\nu}\left(\frac{\pi c}{h}\right) \exp \left(-\frac{2 \pi c}{h}\right)|z|^{2 \nu+1}\{1+($ term vanishing as $|z| \rightarrow \infty)\}$, on $C_{2}, C_{5}$, where $\kappa_{\nu}$ is given in Lemma 2.2. On the other hand, from (2.6) and Lemma 2.1 , for arbitrary $\varepsilon>0$, we have

$$
\left|\Phi_{\nu h}(z)\right| \leqq 2 \pi(1+\varepsilon)|z|^{2 \nu+1} \exp \left(-\frac{2 \pi}{h}|\operatorname{Im} z|\right) \leqq 2 \pi(1+\varepsilon)|z|^{2 \nu+1}
$$

on $C_{6}+C_{1}$ and $C_{3}+C_{4}$ if $N_{1}$ and $N_{2}$ are sufficiently large. Then we have

$$
\begin{aligned}
& \left|\frac{1}{2 \pi \mathrm{i}} \int_{C_{1}+\cdots+C_{6}} z^{2 \nu+1} f(z) \Phi_{\nu h}(z) \mathrm{d} z\right| \\
\leqq & \widetilde{C}_{\nu c} \exp \left(-\frac{2 \pi c}{h}\right)\left(\int_{C_{2}}+\int_{C_{5}}\right)|z|^{2 \nu+1}|f(z)||\mathrm{d} z| \\
& +(1+\varepsilon)\left(\int_{C_{3}+C_{4}}+\int_{C_{6}+C_{1}}\right)|z|^{2 \nu+1}|f(z)||\mathrm{d} z|,
\end{aligned}
$$

where $\widetilde{C}_{\nu c}$ is a positive constant depending only on $\nu$ and $d$. The integrals of the second term on the right-hand side vanish as $N_{1}, N_{2} \rightarrow \infty$ because of (2.1). Therefore, taking the limit $c \uparrow d$, we obtain the inequality (2.4).

We now compare the claim of Theorem 2.1 with the one on the quadrature error of the trapezoidal formula (1.5) (Theorem 3.2.1 in [10]), namely, 
Theorem 2.2. $\quad$ Let $h>0$ and $f(z) \in \mathcal{B}(-1 / 2, d)$. Then an upper bound for the quadrature error of the trapezoidal formula (1.5) is given by the inequality

$$
\left|\int_{-\infty}^{\infty} f(x) \mathrm{d} x-h \sum_{k=-\infty}^{\infty} f(k h)\right| \leqq \frac{\exp (-2 \pi d / h)}{1-\mathrm{e}^{-2 \pi d / h}} \mathcal{N}_{-1 / 2 d}(f) .
$$

Theorem 2.2 says that the quadrature error of the trapezoidal formula (1.5) for a function $f(x)$ decays exponentially with order $\mathrm{O}[\exp (-2 \pi d / h)]$ as a function of the node density if $f(z) \in \mathcal{B}(-1 / 2, d)$. Therefore, if $f(z) \in \mathcal{B}(\nu, d)$ $(\nu \geqq-1 / 2)^{4}$, the quadrature errors of the formula (1.1) and the trapezoidal one (1.5) are of the same order. This coincidence of the error orders seems natural if we remark that the trapezoidal formula is a special case of the quadrature one (1.1). In fact, if we put $\nu=-1 / 2$ in (1.1), we obtain from $J_{-1 / 2}(z)=$ $\sqrt{2 /(\pi z)} \cos z$ that

$$
\int_{-\infty}^{\infty} f(x) \mathrm{d} x \approx h \sum_{k=-\infty}^{\infty} f\left(h\left(k+\frac{1}{2}\right)\right),
$$

which is nothing but the (midpoint) trapezoidal formula.

Lemmas Used in the Proofs of Theorem 2.1, 3.1. We here present the lemmas used in the proof of Theorem 2.1, 3.1 and the proofs of these lemmas.

Lemma 2.1. $\quad$ For arbitrary $\varepsilon>0$, there exists a positive integer $N$ such that

$$
\frac{1}{\left|J_{\nu}(z)\right|} \leqq(1+\varepsilon) \sqrt{2 \pi|z|} \mathrm{e}^{-|\operatorname{Im} z|} \quad \text { if }|\operatorname{Re} z|=\pi\left(N+\frac{\nu}{2}+\frac{1}{4}\right)
$$

Proof. It is sufficient to prove the lemma in the case that $\operatorname{Re} z>0$ since we have $\left|J_{\nu}\left(\mathrm{e}^{ \pm \mathrm{i} \pi} z\right)\right|=\left|\mathrm{e}^{ \pm \mathrm{i} \nu \pi} J_{\nu}(z)\right|=\left|J_{\nu}(z)\right|$. The asymptotic expansion of $\left|J_{\nu}(z)\right|(\S 7.2$ in $[13])$

$$
\begin{array}{r}
J_{\nu}(z)=\frac{1}{\sqrt{2 \pi z}}\left[\mathrm{e}^{\mathrm{i}(z-\nu \pi / 2-\pi / 4)}\left\{1+\mathrm{O}\left(z^{-1}\right)\right\}+\mathrm{e}^{-\mathrm{i}(z-\nu \pi / 2-\pi / 4)}\left\{1+\mathrm{O}\left(z^{-1}\right)\right\}\right] \\
\text { as } z \rightarrow \infty,|\arg z|<\pi
\end{array}
$$

\footnotetext{
${ }^{4}$ We here remark that $\mathcal{B}(\nu, d) \subset \mathcal{B}\left(\nu^{\prime}, d\right)$ if $\nu>\nu^{\prime}(>-1)$.
} 
leads us to the fact that, for arbitrary $\varepsilon>0$, there exists a positive integer $N>0$ such that, if $\operatorname{Re} z=\pi(N+\nu / 2+1 / 4)$,

$$
\left|J_{\nu}(z)\right| \geqq\left|\frac{1}{\sqrt{2 \pi|z|}}\left[(-1)^{N} \mathrm{e}^{-\operatorname{Im} z} \frac{1}{1+\varepsilon}+(-1)^{N} \mathrm{e}^{\operatorname{Im} z} \frac{1}{1+\varepsilon}\right]\right| \geqq \frac{\mathrm{e}^{\operatorname{Im} z}}{\sqrt{2 \pi|z|}} \frac{1}{1+\varepsilon} .
$$

This is nothing but the inequality (2.14).

Lemma 2.2. $\quad$ For arbitrary $M>0$, we define the value $\kappa_{\nu}(M)$ by

$$
\kappa_{\nu}(M)=\sup _{|\operatorname{Im} z| \geqq M}\left\{\frac{\mathrm{e}^{|\operatorname{Im} z|}}{\sqrt{2 \pi|z|}\left|J_{\nu}(z)\right|}\right\} .
$$

Then the value $\kappa_{\nu}(M)$ satisfies the properties that $(\mathrm{i}) \kappa_{\nu}(M)$ decreases monotonously in $M$, (ii) $\kappa_{\nu}(M)<+\infty$ and (iii) $\lim _{M \rightarrow \infty} \kappa_{\nu}(M)=1$.

Using $\kappa_{\nu}(M)$ defined in the above lemma, we have the inequality

$$
\frac{1}{\left|J_{\nu}(z)\right|} \leqq \kappa_{\nu}(M) \sqrt{2 \pi|z|} \mathrm{e}^{-|\operatorname{Im} z|} \quad \text { if }|\operatorname{Im} z| \geqq M
$$

for arbitrary $M>0$.

Proof. The property (i) is obvious. In order to prove the properties (ii) and (iii), we rewrite $\kappa_{\nu}(M)$ as

$$
\kappa_{\nu}(M)=\sup _{|\operatorname{Im} z| \geqq M, \operatorname{Re} z \geqq 0}\left\{\frac{\mathrm{e}^{|\operatorname{Im} z|}}{\sqrt{2 \pi|z|}\left|J_{\nu}(z)\right|}\right\},
$$

which is guaranteed by the relation $\left|J_{\nu}\left(\mathrm{e}^{ \pm \mathrm{i} \pi} z\right)\right|=\left|\mathrm{e}^{ \pm \mathrm{i} \nu \pi} J_{\nu}(z)\right|=\left|J_{\nu}(z)\right|$. From the above expression of $\kappa_{\nu}(M)$ and the asymptotic expansion (2.15), we obtain the property (iii).

It is easy to prove the property (ii). In fact, from the asymptotic expansion (2.15), we have for sufficiently large $R>0$

$$
\frac{\mathrm{e}^{|\operatorname{Im} z|}}{\sqrt{2 \pi|z|}\left|J_{\nu}(z)\right|} \approx 1 \quad \text { if }|z|>R,|\operatorname{Im} z| \geqq M, \operatorname{Re} z \geqq 0
$$

and, in addition, we have

$$
\sup \left\{\frac{\mathrm{e}^{|\operatorname{Im} z|}}{\sqrt{2 \pi|z|}\left|J_{\nu}(z)\right|}|| z|\leqq R,| \operatorname{Im} z \mid \geqq M, \operatorname{Re} z \geqq 0\right\}<\infty
$$

since $J_{\nu}(z)$ has zeros only on the real axis. 


\section{$\S 3 . \quad$ Quadrature Formula of Interpolation-Type}

We here show that the quadrature formula (1.1) can be regarded as of interpolation-type. In fact, it can be obtained by integrating formally the following interpolation formula with the same nodes, namely,

$$
\begin{aligned}
f(x) \approx \mathcal{L}_{\nu h} f(x)= & -\sum_{\substack{k=-\infty \\
k \neq 0}}^{\infty} f\left(h \xi_{\nu k}\right) \frac{\left(h \xi_{\nu k} / x\right)^{\nu} J_{\nu}(\pi x / h)}{J_{\nu+1}\left(\pi \xi_{\nu k}\right)\left(\pi x / h-\pi \xi_{\nu k}\right)} \\
= & -\sum_{k=1}^{\infty} f\left(h \xi_{\nu k}\right) \frac{\left(h \xi_{\nu k} / x\right)^{\nu} J_{\nu}(\pi x / h)}{J_{\nu+1}\left(\pi \xi_{\nu k}\right)\left(\pi x / h-\pi \xi_{\nu k}\right)} \\
& +\sum_{k=1}^{\infty} f\left(-h \xi_{\nu k}\right) \frac{\left(h \xi_{\nu k} / x\right)^{\nu} J_{\nu}(\pi x / h)}{J_{\nu+1}\left(\pi \xi_{\nu k}\right)\left(\pi x / h+\pi \xi_{\nu k}\right)}
\end{aligned}
$$

with a real constant $\nu$. It is easily shown by $J_{\nu}^{\prime}\left(\pi \xi_{\nu k}\right)=-J_{\nu+1}\left(\pi \xi_{\nu k}\right)$, which is obtained from the formula (2.8), that

$$
\mathcal{L}_{\nu h} f\left(h \xi_{\nu k}\right)=f\left(h \xi_{\nu k}\right) . \quad k= \pm 1, \pm 2, \ldots
$$

If $-1<\nu<1 / 2$, we can formally integrate (3.1) term by term to obtain

$$
\begin{aligned}
\mathcal{I}_{\nu}(f) \approx & \int_{-\infty}^{\infty}|x|^{2 \nu+1} \mathcal{L}_{\nu h} f(x) \mathrm{d} x \\
= & -\sum_{k=1}^{\infty} f\left(h \xi_{\nu k}\right) \frac{\left(h \xi_{\nu k}\right)^{\nu}}{J_{\nu+1}\left(\pi \xi_{\nu k}\right)} \int_{-\infty}^{\infty} \frac{|x|^{2 \nu+1} x^{-\nu} J_{\nu}(\pi x / h)}{\pi x / h-\pi \xi_{\nu k}} \mathrm{~d} x \\
& +\sum_{k=1}^{\infty} f\left(-h \xi_{\nu k}\right) \frac{\left(h \xi_{\nu k}\right)^{\nu}}{J_{\nu+1}\left(\pi \xi_{\nu k}\right)} \int_{-\infty}^{\infty} \frac{|x|^{2 \nu+1} x^{-\nu} J_{\nu}(\pi x / h)}{\pi x / h+\pi \xi_{\nu k}} \mathrm{~d} x .
\end{aligned}
$$

Then we obtain the quadrature formula (1.1) using the formula

$$
\int_{-\infty}^{\infty} \frac{|x|^{2 \nu+1} x^{-\nu} J_{\nu}(\pi x / h)}{\pi x / h-\pi \xi_{\nu k}} \mathrm{~d} x=-\operatorname{sgn} k\left(h \xi_{\nu|k|}\right)^{\nu+1} Y_{\nu}\left(\pi \xi_{\nu|k|}\right)
$$

with

$$
\operatorname{sgn} k= \begin{cases}+1 & \text { if } k>0 \\ -1 & \text { if } k<0\end{cases}
$$

if $-1<\nu<1 / 2$. The proof of (3.3) will be given at the end of this section. 
Remark 1. The interpolation formula (3.1) can be obtained by an analogue of the Lagrange interpolation of nodes $x_{1}, x_{2}, \ldots, x_{N}$

$$
f(x) \approx L_{N} f(x)=\sum_{k=1}^{N} f\left(x_{k}\right) \frac{W(x)}{W^{\prime}\left(x_{k}\right)\left(x-x_{k}\right)}
$$

with $W(x)=\prod_{k=1}^{N}\left(x-x_{k}\right)$. In fact, we put

$$
W(x)=\left(\frac{\pi x}{h}\right)^{-\nu} J_{\nu}\left(\frac{\pi x}{h}\right)=\frac{1}{2^{\nu} \Gamma(\nu+1)} \prod_{k=1}^{\infty}\left\{1-\left(\frac{x}{h \xi_{\nu k}}\right)^{2}\right\}
$$

(and $N=\infty$ ) in $(3.4)^{5}$ and, using (2.8), we obtain the interpolation formula (3.1).

Remark 2. In the case of $\nu=-1 / 2$, the interpolation formula (3.1) becomes the Sinc approximation

$$
f(x) \approx \sum_{k=-\infty}^{\infty} f(k h) \frac{\sin [(\pi / h)(x-k h)]}{(\pi / h)(x-k h)},
$$

which is recently applied to various subjects of numerical analysis by Stenger and others [10]. In fact, if we put $\nu=-1 / 2$ in (3.1) and remark that $J_{-1 / 2}(x)=$ $\sqrt{2 /(\pi x)} \cos x$ obtained from formulae 10.1 .1 and 10.1.12 in [1], we have

$$
\mathcal{L}_{-1 / 2 h} f(x)=\sum_{k=-\infty}^{\infty} f\left(h\left(k+\frac{1}{2}\right)\right) \frac{\sin [\pi x / h-\pi(k+1 / 2)]}{\pi x / h-\pi(k+1 / 2)},
$$

which is nothing but the (midpoint) Sinc approximation.

Error Analysis. An upper bound of the interpolation error of (3.1) is given by the following theorem.

Theorem 3.1. Let $\nu>-3 / 2$ and $f(z) \in \mathcal{B}(\nu / 2-1 / 4, d)$. Then the interpolation error of the formula (3.1) is expressed by the complex integral

$$
f(x)-L_{\nu h} f(x)=\frac{J_{\nu}(\pi x / h)}{2 \pi \mathrm{i} x^{\nu}} \int_{\Gamma_{d-0}} \frac{z^{\nu} f(z)}{(z-x) J_{\nu}(\pi z / h)} \mathrm{d} z,
$$

where $x$ is an arbitrary real number, and an upper bound of the error is given by the inequality

$$
\sup _{-\infty<x<\infty}\left|f(x)-L_{\nu h} f(x)\right| \leqq C_{\nu d} \mathcal{N}_{\nu d-0}(f) h^{-\nu-1 / 2} \exp \left(-\frac{\pi d}{h}\right),
$$

where $C_{\nu d}$ is a positive constant depending only on $\nu$ and $d$.

\footnotetext{
${ }^{5}$ The expression of (3.5) can be obtained if we remark that the function $x^{-\nu} J_{\nu}(z)$ is entire with zeros only at $z=\pi \xi_{\nu k}, k= \pm 1, \pm 2, \ldots[13]$.
} 
Proof. We here consider the complex integral

$$
\frac{J_{\nu}(\pi x / h)}{2 \pi \mathrm{i} x^{\nu}} \int_{C_{1}+C_{2}+C_{3}+C_{4}+C_{5}+C_{6}} \frac{z^{\nu} f(z)}{(z-x) J_{\nu}(\pi z / h)} \mathrm{d} z
$$

where $N_{1}, N_{2}$ are positive numbers and $C_{1}, C_{2}, \ldots, C_{6}$ are rectilinear lines given in Figure 1). Since $f(z)$ is analytic in the domain $D_{d}$ and that $z^{-\nu} J_{\nu}(\pi z / h)$ is entire function with zeros only at $z=h \xi_{\nu k}, k= \pm 1, \pm 2, \ldots$, we obtain by the residue theorem the equation

$$
\text { integral }(3.9)=f(x)+\sum_{\substack{k=-N_{2} \\ k \neq 0}}^{N_{1}} f\left(h \xi_{\nu k}\right) \frac{\left(h \xi_{\nu k} / x\right)^{\nu} J_{\nu}(\pi x / h)}{J_{\nu+1}\left(\pi \xi_{\nu k}\right)\left(\pi x / h-\pi \xi_{\nu k}\right)}
$$

where we used the formula (2.8). By (2.1) and Lemma 2.1, the integrals on $C_{6}+C_{1}$ and $C_{3}+C_{4}$ in (3.9) vanish as $N_{1}, N_{2} \rightarrow \infty$. Therefore we have (3.7) by taking the limit $c \uparrow d$.

The inequality (3.8) is obtained by estimating the absolute value of the right hand side of (3.7) as in the proof of Theorem 2.1. Using Lemma 2.1 and 2.2 , we have for arbitrary $\varepsilon>0$

$$
\begin{gathered}
\left|\int_{C_{1}+\cdots}+C_{6} \frac{z^{\nu} f(z) \mathrm{d} z}{(z-x) J_{\nu}(\pi z / h)}\right| \leqq \kappa_{\nu}\left(\frac{\pi c}{h}\right) \sqrt{\frac{2 \pi^{2}}{h}} \frac{\mathrm{e}^{-\pi c / h}}{c}\left(\int_{C_{2}}+\int_{C_{5}}\right)|z|^{\nu+1 / 2} \mid \\
\times\left. f(z)|| \mathrm{d} z\left|+\sqrt{\frac{2 \pi^{2}}{h}} \frac{2(1+\varepsilon)}{R-|x|}\left(\int_{C_{3}+C_{4}}+\int_{C_{6}+C_{1}}\right)\right| z\right|^{\nu+1 / 2}|f(z)||\mathrm{d} z|
\end{gathered}
$$

with $R=\min \left\{R_{1}, R_{2}\right\}$ if $N_{1}$ and $N_{2}$ are sufficiently large. The integrals of the second term on the right-hand side vanish as $N_{1}, N_{2} \rightarrow \infty$ because of (2.1). Therefore, using the inequality $([13], \S 3.31)\left|x^{-\nu} J_{\nu}(\pi x / h)\right| \leqq(\pi /(2 h))^{\nu} / \Gamma(\nu+$ $1)$, we obtain the inequality (3.8).

We here compare the interpolation error given in Theorem 3.1 with the quadrature error given in Theorem 2.1. From the two theorems, we have for functions $f(z) \in B(\nu, d)(-1<\nu<1 / 2)$

$$
\left(\begin{array}{c}
\text { interpolation } \\
\text { error }
\end{array}\right)=\mathrm{O}\left[\exp \left(-\frac{\pi d}{h}\right)\right],\left(\begin{array}{c}
\text { quadrature } \\
\text { error }
\end{array}\right)=\mathrm{O}\left[\exp \left(-\frac{2 \pi d}{h}\right)\right] \text {, }
$$

that is,

$$
\text { (quadrature error }) \approx(\text { interpolation error })^{2} \text {. }
$$


In other words, the accuracy of the quadrature formula doubles the one of the corresponding interpolation formula. We remark that the Gauss-type quadrature formula and the trapezoidal one (1.5) have the same property. In fact, the $n$-point Gauss-type quadrature formulae give the exact integral values for polynomial of degree $<2 n-1$ while the $n$-point orthogonal polynomial interpolation formulae, from which the Gauss-type quadrature ones are obtained by term-by-term integration, give the exact function value for polynomials of degree $<n$. In the case of the trapezoidal formula, the quadrature error is of order $\mathrm{O}[\exp (-2 \pi d / h)]$ for integrand functions $f(z) \in \mathcal{B}(-1 / 2, d)$ as given in Theorem 2.2 while the error of the corresponding interpolation formula, namely, the Sinc approximation (3.6), from which the trapezoidal one is obtained by term-byterm integration, is of order $\mathrm{O}[\exp (-\pi d / h)]$ for functions $f(z) \in \mathcal{B}(-1 / 2, d)$ (Theorem 3.1.3 in [10]).

Proof of (3.3). We here prove the formula (3.3).

Proof. Note that we have

$$
\begin{aligned}
& \int_{-\infty}^{\infty} \frac{|x|^{2 \nu+1} x^{-\nu} J_{\nu}(\pi x / h)}{\pi x / h-\pi \xi_{\nu k}} \mathrm{~d} x \\
& \quad=\left(\frac{h}{\pi}\right)^{\nu+1}\left\{\int_{0}^{\infty} \frac{x^{\nu+1} J_{\nu}(x)}{x-\pi \xi_{\nu k}} \mathrm{~d} x-\int_{0}^{\infty} \frac{x^{\nu+1} J_{\nu}(x)}{x+\pi \xi_{\nu k}} \mathrm{~d} x\right\}
\end{aligned}
$$

since $x^{-\nu} J_{\nu}(\pi x / h)$ is an even function.

We here consider the complex integral

$$
\int_{C} \frac{z^{\nu+1} H_{\nu}^{(1)}(z)}{z-\pi \xi_{\nu k}} \mathrm{~d} z=0,
$$

where $C$ is the integral path given in Figure 2 and the equality is evident from Cauchy's theorem. From the asymptotic expansions of $H_{\nu}^{(1)}(z)(2.6)$, there exists $C>0$ such that $\left|H_{\nu}^{(1)}(z)\right| \leqq C \sqrt{2 /(\pi|z|)} \exp (-\operatorname{Im} z)$ for sufficiently large $|z|(0 \leqq \arg z \leqq \pi)$. Then we have the upper bounds of the integrals on $C_{1}, C_{2}$ and $C_{3}$

$$
\begin{gathered}
\mid\left(\text { integral on } C_{1}\right) \mid \leqq C \sqrt{\frac{2}{\pi R_{1}}} \int_{0}^{c} \mathrm{e}^{-y} \mathrm{~d} y \leqq C \sqrt{\frac{2}{\pi R_{1}}}, \\
\mid\left(\text { integral on } C_{2}\right)\left|\leqq C \sqrt{\frac{2}{\pi c}}\left(R_{1}+R_{2}\right) \mathrm{e}^{-c} . \quad\right|\left(\text { integral on } C_{3}\right) \mid \leqq C \sqrt{\frac{2}{\pi R_{2}}},
\end{gathered}
$$




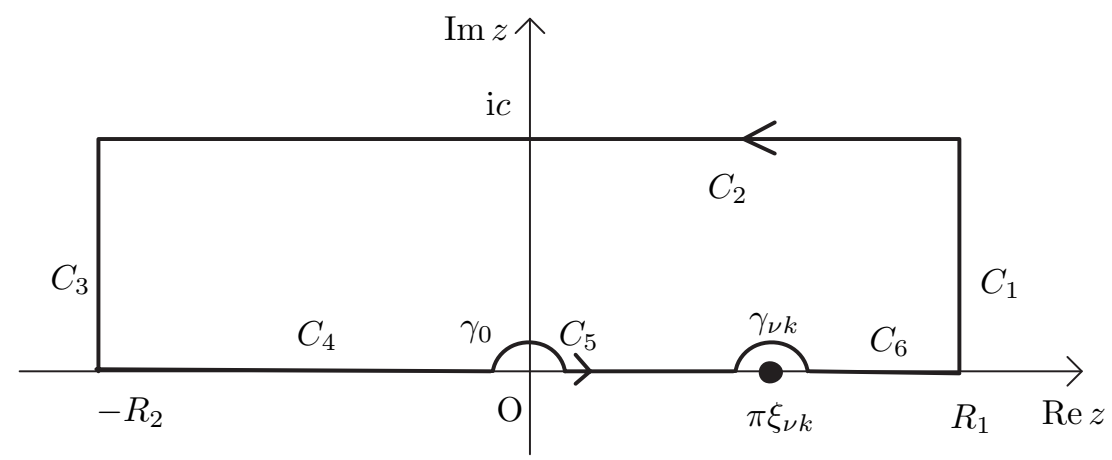

Figure 2. The integral path $C=C_{1}+C_{2}+C_{3}+C_{4}+\gamma_{0}+C_{5}+\gamma_{\nu k}+C_{6}$ for the proof of (3.3), where $R_{1}, R_{2}, c$ are positive constants, $\gamma_{\nu k}$ is the semi-circle of radius $\rho>0$ with centre at $z=h \xi_{\nu k}$ and $\gamma_{0}$ is the semi-circle of radius $\rho$ with centre at $z=0$.

The integral on $\gamma_{\nu k}$ is computed as follows. If $k>0$, we have

$$
\begin{aligned}
\left(\text { integral on } \gamma_{\nu k}\right) & =-\mathrm{i} \pi \times\left(\text { residue at } z=\pi \xi_{\nu k}\right)+\mathrm{O}(\rho) & \\
& =\pi\left(\pi \xi_{\nu k}\right)^{\nu+1} Y_{\nu}\left(\pi \xi_{\nu k}\right)+\mathrm{O}(\rho) & \text { as } \rho \downarrow 0
\end{aligned}
$$

and, if $k<0$,

$$
\begin{aligned}
\text { (integral on } \left.\gamma_{\nu k}\right) & =-\mathrm{i} \pi \times\left(\text { residue at } z=\mathrm{e}^{\mathrm{i} \pi} \pi \xi_{\nu|k|}\right)+\mathrm{O}(\rho) \\
& =-\mathrm{i} \pi\left(\mathrm{e}^{\mathrm{i} \pi} \pi \xi_{\nu|k|}\right)^{\nu+1} H_{\nu}^{(1)}\left(\mathrm{e}^{\mathrm{i} \pi} \pi \xi_{\nu|k|}\right)+\mathrm{O}(\rho) \\
& =-\pi\left(\pi \xi_{\nu|k|}\right)^{\nu+1} Y_{\nu}\left(\pi \xi_{\nu|k|}\right)+\mathrm{O}(\rho) \quad \text { as } \rho \downarrow 0,
\end{aligned}
$$

where we used (2.9) on the third equality. The integral on $\gamma_{0}$ is shown to vanish as $\rho \downarrow 0$ by a way similar to the one in the proof of Theorem 2.1. The limit of the integral on $C_{4}+C_{5}+C_{6}$ as $\rho \downarrow 0$ is shown to be

$$
\begin{aligned}
& \left.\lim _{\rho \downarrow 0} \text { (integral on } C_{4}+C_{5}+C_{6}\right) \\
= & \text { p.v. } \int_{0}^{R_{1}} \frac{x^{\nu+1} H_{\nu}^{(1)}(x)}{x-\pi \xi_{\nu k}} \mathrm{~d} x-\text { p.v. } \int_{0}^{R_{2}} \frac{\left(\mathrm{e}^{\mathrm{i} \pi} x\right)^{\nu+1} H_{\nu}^{(1)}\left(\mathrm{e}^{\mathrm{i} \pi} x\right)}{x+\pi \xi_{\nu k}} \\
= & \text { p.v. } \int_{0}^{R_{1}} \frac{x^{\nu+1} H_{\nu}^{(1)}(x)}{x-\pi \xi_{\nu k}} \mathrm{~d} x-\text { p.v. } \int_{0}^{R_{2}} \frac{x^{\nu+1} H_{\nu}^{(2)}(x)}{x+\pi \xi_{\nu k}} \mathrm{~d} x,
\end{aligned}
$$

where we used (2.9) on the second equality. 
Substituting the above results into (3.13), we have

$$
\begin{aligned}
0 & =\lim _{R_{1}, R_{2} \rightarrow \infty \rho \downarrow 0} \lim _{\rho \downarrow} \operatorname{Re} \frac{z^{\nu+1} H_{\nu}^{(1)}(z)}{z-\pi \xi_{\nu k}} \mathrm{~d} z \\
& =\int_{0}^{\infty} \frac{x^{\nu+1} J_{\nu}(x)}{x-\pi \xi_{\nu k}} \mathrm{~d} x-\int_{0}^{\infty} \frac{x^{\nu+1} J_{\nu}(x)}{x+\pi \xi_{\nu k}} \mathrm{~d} x+\operatorname{sgn} k \pi\left(\pi \xi_{\nu|k|}\right)^{\nu+1} Y_{\nu}\left(\pi \xi_{\nu|k|}\right) .
\end{aligned}
$$

Consequently, we have (3.3) by (3.12).

\section{§4. Numerical Examples}

We here show examples for the quadrature formula (1.1). All the computations in this paper were carried out on a SUN Blade 150 workstation using programs coded in $\mathrm{C}++$ with double precision working.

Example 1. The integrand function of the first example is

$$
f_{1}(x)=\frac{\exp (-\cosh x)}{1+x^{2}}
$$

which decays double exponentially at infinity. The integrals of functions decaying more slowly are transformed to the integrals of functions decaying double exponentially by the DE transforms used in the DE rules [11]. The exact integral value ${ }^{6}$ of $f_{1}(x)$ with $\nu=0$ is $\mathcal{I}_{0}\left(f_{1}\right)=0.306354694925705 \ldots$. We computed the integral $\mathcal{I}_{0}\left(f_{1}\right)$ by the quadrature formula (1.1) and plotted the relative error of the quadrature $\left|\mathcal{I}_{\nu h}\left(f_{1}\right)-\mathcal{I}_{\nu}\left(f_{1}\right)\right| /\left|\mathcal{I}_{\nu}\left(f_{1}\right)\right|$ as functions of the node density $1 / h$ in Figure $3(\mathrm{a})$, which also includes the interpolation error ${ }^{7}$

$$
\Delta \mathcal{L}_{\nu h}(f) \equiv \sup _{-\infty<x<+\infty}\left|f(x)-\mathcal{L}_{\nu h} f(x)\right| .
$$

The orders of the quadrature and the interpolation errors theoretically estimated in Section 2, 3 or from the numerical results are summarised in Table 1. In the table, the value $d$ in the theoretical error estimates is taken as $d \approx 1$ because $f(z)$ has poles at $z= \pm \mathrm{i}$, and the orders of the errors in the numerical examples are given as functions of $1 / h$ in the form $\exp (-c / h)$ with constants $c(>0)$ estimated by applying the least square fitting to the numerical data.

\footnotetext{
${ }^{6}$ They are obtained by splitting them into two parts at $x=0$, namely, $\mathcal{I}_{\nu}\left(f_{1}\right)=$ $\left(\int_{-\infty}^{0}+\int_{0}^{+\infty}\right)|x|^{2 \nu+1} f_{1}(x) \mathrm{d} x$ and applying the DE formula to these integrals [12] using a program made by Dr. T. Ooura [9].

${ }^{7}$ Actually, the supremum on $(-\infty,+\infty)$ is approximated by the maximum over 1028 points on the interval $[-M, M](M=4.0)$ distributed by using uniform random numbers.
} 


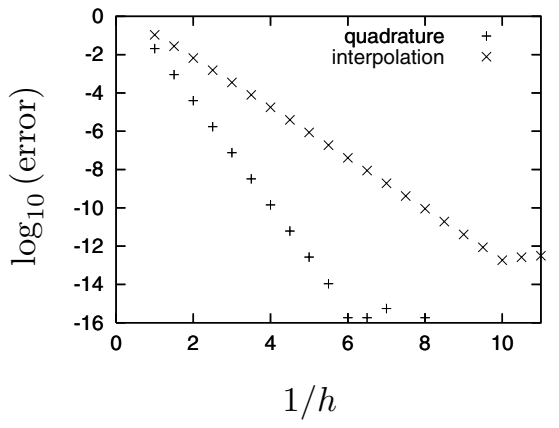

(a) Example 1

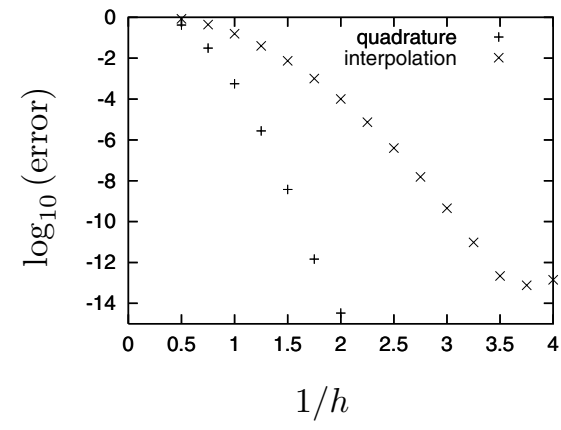

(b) Example 2

Figure 3. The errors of the quadrature formula (1.1) and the interpolation formula (3.1) with $\nu=0$ for (a) $f_{1}(x)$ of Example 1 and for (b) $f_{2}(x)$ of Example 2.

From the figure and the table, it is evident that the numerical results support the results of the theoretical error analysis, that is, the facts that the quadrature error is of order $\mathrm{O}[\exp (-2 \pi d / h)]$ and the relation

$$
\text { (quadrature error })=(\text { interpolation error })^{2} \text {. }
$$

Example 2. The integrand function of the second example is

$$
f_{2}(x)=\exp \left(-x^{2}\right) .
$$

The exact integral value of $f_{2}(x)$ with $\nu=0$ is $\mathcal{I}_{0}\left(f_{1}\right)=1$.

The errors of the quadrature formula (1.1) and the interpolation formula (3.1) are shown as a function of $1 / h$ in Figure $3(b)$. From this figure, we find that the orders of the errors are not in the form $\mathrm{O}[\exp (-c / h)]$ with constants $c(>0)$. The theoretical error estimates by (2.4) and (3.8) are too rough for the function $f_{2}(x)$ and estimating the errors by applying the saddle point method to the complex integrals (2.2) and (3.7) gives more accurate error estimates, which are of the form $\mathrm{O}\left[\exp \left(-c / h^{2}\right)\right]$ with constants $c>0$. The orders of the quadrature and interpolation errors theoretically estimated or from the numerical results are summarized in Table 1 . In the table, the orders of the errors from the numerical results are given in the form $\mathrm{O}[\exp (-c / h)]$ with constants $c(>0)$ estimated by applying the least square fitting to the numerical data. From the table, it is evident that the numerical results make good agreements with the results of the theoretical error analysis. 
Table 1. The quadrature and the interpolation errors for $f_{1}(x)$ of Example 1 and for $f_{2}(x)$ of Example 2 .

\begin{tabular}{cclc}
\hline \multirow{2}{*}{ example } & & \multicolumn{2}{c}{ error } \\
\cline { 3 - 4 } 1 & quadrature & $\begin{array}{l}\mathrm{O}[\exp (-2 \pi d / h)] \\
=\mathrm{O}[\exp (-6.3 \ldots / h)]\end{array}$ & $\mathrm{O}[\exp (-6.4 \ldots / h)]$ \\
& \multirow{2}{*}{ interpolation $[\exp (-\pi d / h)]$} & $\mathrm{O}[\exp (-3.0 \ldots / h)]$ \\
& & $\mathrm{O}[\exp (-3.1 \ldots / h)]$ & \\
\hline \multirow{2}{*}{2} & quadrature & $\mathrm{O}\left[\exp \left(-\pi^{2} / h^{2}\right)\right]$ & $\mathrm{O}\left[\exp \left(-8.9 \ldots / h^{2}\right)\right]$ \\
& & $\mathrm{O}\left[\exp \left(-9.9 \ldots / h^{2}\right)\right]$ & \\
& interpolation $\left[\exp \left(-\pi^{2} /\left(4 h^{2}\right)\right)\right]$ & $\mathrm{O}\left[\exp \left(-2.3 \ldots / h^{2}\right)\right]$ \\
& $=\mathrm{O}\left[\exp \left(-2.5 \ldots / h^{2}\right)\right]$ & \\
\hline
\end{tabular}

\section{$\S 5$. Application to Integrals of the Hankel Transformation Type}

In this section, we present an application of the quadrature formula (1.1) to the computations of (1.4), which was the motivation of the presented study. We here construct a DE-type quadrature formula for (1.4) similar to Ooura and Mori's DE formula for integrals (1.3), namely, a quadrature formula with a DE-type transform such that the nodes approach to the zeros of $J_{\nu}(x)$ double exponentially.

We apply the variable transform

$$
x=\frac{\pi}{h} \psi(t) \quad \text { with } \quad \psi(t)=t \tanh \left(\frac{\pi}{2} \sinh t\right)
$$

to an integral (1.4) and, remarking that the transformed integrand function $\frac{\pi}{h} f\left(\frac{\pi}{h} \psi(t)\right) J_{\nu}\left(\frac{\pi}{h} \psi(t)\right) \psi^{\prime}(t)$ is of the form $|t|^{2 \nu+1} \times$ (even function), apply the quadrature formula (1.1) to the transformed integral. Then we have the approximation

$$
\int_{0}^{\infty} f(x) J_{\nu}(x) \mathrm{d} x \approx \pi \sum_{k=1}^{\infty} w_{\nu k} f\left(\frac{\pi}{h} \psi\left(h \xi_{\nu k}\right)\right) J_{\nu}\left(\frac{\pi}{h} \psi\left(h \xi_{\nu k}\right)\right) \psi^{\prime}\left(h \xi_{\nu k}\right) .
$$

The infinite sum on the right hand side of (5.2) can be truncated with a small number of function evaluations since the quadrature nodes approach to the zeros of $J_{\nu}(x)$, that is, $\frac{\pi}{h} \psi\left(h \xi_{\nu k}\right) \sim \pi \xi_{\nu k}$ double exponentially as $k \rightarrow \infty$. Therefore, we expect that we can effectively compute integrals (1.4) by the DE formula (5.2). 
Numerical Examples. We computed the integrals

$$
\text { (i) } \int_{0}^{\infty} J_{0}(x) \mathrm{d} x=1, \quad \text { (ii) } \int_{0}^{\infty} \frac{x J_{0}(x)}{x^{2}+1} \mathrm{~d} x=K_{0}(1),
$$

where $K_{\nu}$ is the modified Bessel function of order $\nu$ of the second kind, by the formula (5.2).

Figure 4 shows the relative errors of the formula (5.2) for the integrals (i) and (ii) as a function of the node density $1 / h$ or the number of function evaluations $N$, the minimal integer $k$ such that

$$
\left|f\left(\frac{\pi}{h} \psi\left(h \xi_{\nu k}\right)\right) J_{\nu}\left(\frac{\pi}{h} \psi\left(h \xi_{\nu k}\right)\right) \psi^{\prime}\left(h \xi_{\nu k}\right)\right|<\varepsilon=10^{-15}
$$

and the infinite sum of (5.2) is truncated at the $k$-term. From Figure 4 , we find that the DE formula (5.2) work well for the integral (i) but not so for the integral (ii).
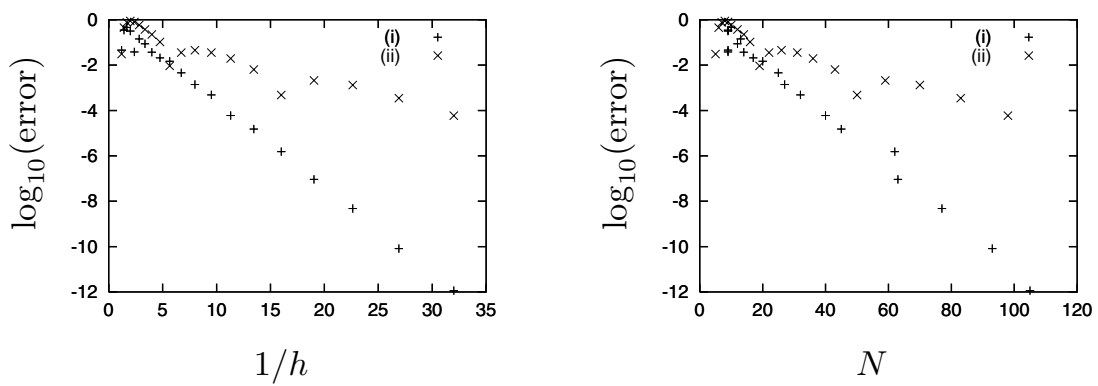

Figure 4. The relative errors of the DE formula (5.2) for the integrals (i) and (ii) as a function of the nodes density $1 / h$ or the number of function evaluations $N$.

The reason why the DE formula fails to compute the integral (ii) efficiently is considered to be as follows. The integrand function of (ii) has singularities at $z= \pm \mathrm{i}$. Then the transformed integrand function $f\left(\frac{\pi}{h} \psi(w)\right) J_{\nu}\left(\frac{\pi}{h} \psi(w)\right) \psi^{\prime}(w)$ has singularities at the images of $z= \pm \mathrm{i}$ by the mapping $w=\psi^{-1}\left(\frac{h}{\pi} z\right)$, which approach the real axis as $h \rightarrow 0$. It is known in theoretical error analysis of numerical integrations [12] that a quadrature formula does not work well if the integrand function has singularities near the real axis. Therefore, the quadrature (5.2) is efficient if $f(x)$ is an entire function, and not so if $f(x)$ has singularities.

A remedy for this weakness is to adopt a DE-type transform such that the singularities of the transformed integrand function does not approach to 
the real axis as $h \rightarrow 0$ as the one used in the robust DE formula for Fourier transformation type integrals by Ooura and Mori [7]. However, it is difficult to find the explicit form of a transform satisfying the above property.

\section{§6. Concluding Remarks}

In this paper, we investigated the quadrature formula (1.1) with the zeros of the Bessel functions as nodes and showed that the formula has the following three nice properties: First, the quadrature formula converges exponentially a function of the density of nodes $1 / h$. Second, the quadrature formula can be obtained by integrating an interpolation formula with the same nodes and that, as the Gauss-type formulae and the trapezoidal one, the accuracy of the quadrature doubles the one of the interpolation. Lastly, we applied the formula to the computations of integrals of the Hankel transform type. In relation to integrals of the Hankel transform type, we also remark that Ooura and Mori have presented DE-type formulae for oscillatory functions with slow decay $[5,6,7]$ in addition to their first DE-type formula for Fourier integrals [8].

Problems for future studies are to apply the quadrature formula (1.1) or the interpolation formula (3.1) to practical computations and to improve the DE formula for integrals of the Hankel transform type so that it works well for integrand functions with singularities as mentioned in Section 5. As shown in this paper, the quadrature formula (1.1) has nice properties like the trapezoidal one (1.5), which gives the basis of the $\mathrm{DE}$ formulae together with the $\mathrm{DE}$ transforms. Therefore we expect that the formula can be used in many subjects of numerical computations.

\section{Acknowledgement}

The author is grateful to Prof. M. Sugihara of the University of Tokyo for his help and encouragement throughout this study and to the referees for reading carefully our paper and giving helpful comments.

\section{References}

[1] Abramowitz, M. and Stegun, I. A., (eds.), Handbook of Mathematical Functions with Formulas, Graphs and Mathematical Tables, National Bureau of Standards, Washington, D.C., 1964.

[2] Frappier, C. and Olivier, P., A quadrature formula involving zeros of Bessel functions, Math. Comp., 60 (1993) 303-316. 
[3] Ghanem, R. B., Quadrature formulae using zeros of Bessel functions as nodes, Math. Comp., 221 (1998), 323-336.

[4] Grozev, G. R. and Rahman, Q. I., A quadrature formula with zeros of Bessel functions as nodes, Math. Comp., 64 (1995) 715-725.

[5] Ooura, T., A generalization of the continuous Euler transformation and its application to numerical quadrature, J. Comput. Appl. Math., 157 (2003), 251-259.

[6] A continuous Euler transformation and its applications to the Fourier transforms of a slowly decaying functions, J. Comput. Appl. Math., 130 (2001), 259-270.

[7] Ooura, T. and Mori, M., A robust double exponential formula for Fourier-type integrals, J. Comput. Appl. Math., 112 (1999), 229-241.

[8] The double exponential formula for oscillatory functions over half interval, $J$. Comput. Appl. Math., 38 (1991), 353-360.

[9] Ooura, T., Ooura's Mathematical Software Packages, http://www.kurims.kyoto-u.ac.jp/ ooura/.

[10] Stenger, F., Numerical Methods Based on Sinc and Analytic Functions, Springer-Verlag, New York, 1993.

[11] Takahasi, H. and Mori, M., Double exponential formula for numerical integration, Publ. RIMS, Kyoto Univ., 9 (1974) 121-144.

[12] Error estimation in the numerical integration of analytic functions, Rep. Comp. Centre, Univ. Tokyo, 3 (1970) 41-108.

[13] Watson, G. N., A Treatise on the Theory of Bessel Functions, 2nd ed., Cambridge Univ. Press, London, 1944. 